\title{
Time-dependent orientation coupling in equilibrium polymer melts
}

Article

Published Version

Cao, J. and Likhtman, A. E. (2010) Time-dependent orientation coupling in equilibrium polymer melts. Physical Review Letters, 104 (20). 207801. ISSN 0031-9007 doi: https://doi.org/10.1103/PhysRevLett.104.207801 Available at https://centaur.reading.ac.uk/17181/

It is advisable to refer to the publisher's version if you intend to cite from the work. See Guidance on citing.

Published version at: http://dx.doi.org/10.1103/PhysRevLett.104.207801

To link to this article DOI: http://dx.doi.org/10.1103/PhysRevLett.104.207801

Publisher: American Physical Society

All outputs in CentAUR are protected by Intellectual Property Rights law, including copyright law. Copyright and IPR is retained by the creators or other copyright holders. Terms and conditions for use of this material are defined in the End User Agreement.

\section{www.reading.ac.uk/centaur}

\section{CentAUR}

Central Archive at the University of Reading

Reading's research outputs online 


\title{
Time-Dependent Orientation Coupling in Equilibrium Polymer Melts
}

\author{
Jing Cao and Alexei E. Likhtman* \\ Department of Mathematics, University of Reading, Whiteknights, Reading RG6 6AX, United Kingdom
} (Received 18 December 2009; revised manuscript received 29 March 2010; published 20 May 2010)

\begin{abstract}
The motion in concentrated polymer systems is described by either the Rouse or the reptation model, which both assume that the relaxation of each polymer chain is independent of the surrounding chains. This, however, is in contradiction with several experiments. In this Letter, we propose a universal description of orientation coupling in polymer melts in terms of the time-dependent coupling parameter $\kappa(t)$. We use molecular dynamics simulations to show that the coupling parameter increases with time, reaching about 50\% at long times, independently of the chain length or blend composition. This leads to predictions of component dynamics in mixtures of different molecular weights from the knowledge of monodisperse dynamics for unentangled melts. Finally, we demonstrate that entanglements do not play a significant role in the observed coupling.
\end{abstract}

Slow relaxation in polymer melts has attracted the constant attention of theoreticians for the last 40 years, perhaps partly because it is still lacking a general framework description. Indeed, melts of short chains are called unentangled and described by the Rouse theory [1]. Relaxation in melts of longer chains is believed to be dominated by entanglements, and is consequently described by the tube theory [2]. However, both theories rely on a very simplistic assumption, namely, that all chains move independently from each other; i.e., the effect of all other chains on a probe chain can be described by random delta-correlated forces and by the static tube constraints for longer chains. The reasoning behind such an assumption is based on the Flory theorem, which states that excluded volume interaction in polymer melts are screened. This makes the chains obey Gaussian random walk statistics on large scales, making single chain dynamics Rouse-like at large scales. However, the fact that each individual chain obeys Rouse dynamics does not mean that the total stress relaxation is also known: this will only be true if cross-correlation between the chains is negligible, which as we show below is clearly not the case.

The tube theory uses an assumption of chain independence twice: each chain reptates in its tube independently, and the piece of chain, which escaped from the tube, is assumed to adopt a random orientation independent of the surrounding chains. Merrill et al. [3] demonstrated a longtime ago that if the second assumption is violated, the relaxation will be slower than expected from the tube theory. Thus, the validity of both Rouse and the tube theory clearly depends on the coupling and cross-correlations between orientation relaxation of different chains in the melt, which we observe and discuss in this letter.

Experimentally, orientation coupling was investigated by a combination of dielectric dichroism and birefringence by Ylitalo et al. [4], and by NMR by Graf et al. [5]. In the first group of experiments, one of the components in binary mixture was labeled and its orientation relaxation was measured after step-strain experiment. The labeling was achieved by replacing a portion of the hydrogens on the polymer backbone with deuterium and then performing the measurements at the infrared wavelength of the carbondeuterium vibrational absorption. It was shown that short component orientation is not fully relaxed until the longest relaxation time of the long component, and that the relaxation of short component becomes proportional to the total relaxation after the expected reptation time of the short component: $S_{\text {short }}(t) / S_{\text {short }}(0)=\varepsilon S_{\text {total }}(t) / S_{\text {total }}(0)$, where $S(t)$ is the orientation relaxation function. The coupling parameter $\varepsilon$ defined through this relationship was found to be $\varepsilon \approx 0.45 \pm 0.05$. It was also shown that in order to describe the relaxation with the tube theory, the coupling effects must be taken into account. However, such coupling coefficient can only be measured after relaxation of short component. Also, this definition is not applicable to monodisperse melts. In ${ }^{1} \mathrm{H}$ Double quantum NMR experiments Graf et al. measured the chain dynamics of a polybutadiene melt well above the glass transition and confirmed significant residual bond orientation after elementary entanglement time $\tau_{e}$. They concluded that restrictions by entanglements can not be the only source of orientation coupling. A possible reason for this strongly anisotropic orientational dynamics may be the intermolecular orientational correlations. Our molecular dynamics simulation support this conclusion.

Orientation coupling effects were also investigated in molecular dynamics simulations by Baljon et al. [6], whose protocol was similar to the experimental one. They simulated one very long chain $(N=5000)$ in the sea of small chains after step deformation and looked at the order parameter $q^{\alpha \beta}=(3 / 2)^{1 / 2}\left(\mathbf{u}^{\alpha} \mathbf{u}^{\beta}-\frac{1}{3} \delta^{\alpha \beta}\right)$, where $\mathbf{u}$ is the unit bond vector and $\delta^{\alpha \beta}$ is a unit tensor. Similarly to the experiment of Ylitalo et al. [4], they found that the short chains order parameter does not relax to zero after the relaxation time of short chains, but instead becomes proportional to the order parameter of the long chain, with the 
somewhat smaller proportionality coefficient $\varepsilon \approx 0.28$. Baljon et al. also attempted to calculate $\varepsilon$ from the static properties of the melt, namely, from the orientation distribution function around each bond. The long chain bonds were oriented by using an explicit nematic field instead of stretching. However, the smaller coefficient $\varepsilon \approx 0.18$ was found. They argued that the difference can be explained by positional anisotropy of chain bonds belonging to other chains. By following the same procedure, we were able to reproduce similar results. Thus, a more universal measure of coupling is desirable.

The aim of the present Letter is to investigate coupling effects as a function of time by using equilibrium molecular dynamics simulations, and to develop a universal description of coupling without relying on binary blends or nonequilibrium experiments. We will use a popular Kremer-Grest polymer model [7,8], consisting of purely repulsive Lennard-Jones beads connected by FENE springs (the same model was used by Baljon et al.). Introduction of multiple-tau correlators for each chain allowed very precise calculation of various correlation functions, using all available data at each time step. The details of the simulations are described elsewhere [9]. Most of the simulations were running in the system with monomer number density equal to $0.85 \sigma^{-3}$ where $\sigma$ is the Lennard-Jones length unit. The main tool we will be using are cross-correlation functions between different chains' orientation tensor. The orientation tensor of chain $j$ is defined as $O_{j}^{\alpha \beta}(t)=\sum_{i=1}^{N-1} u_{i j}^{\alpha}(t) u_{i j}^{\beta}(t)$, where $N$ is the number of monomers in chain $j, u_{i j}$ is a bond vector connecting monomers $i$ and $i+1$ of chain $j, \alpha$ and $\beta$ go through Cartesian coordinates $x, y, z$. Since the bond lengths are very quickly equilibrated in our simulations, it does not matter whether $u_{i j}$ is normalized by its length or not. We adopted an unnormalized definition for computational convenience and easier analysis with Rouse modes in the future. Using this definition we can compute a variety of autocorrelation and cross-correlation functions in the melt. But before doing this, let us check if the stressoptical law works in our equilibrium simulations. The stress-optical law states that the relaxation function of total orientation tensor should be proportional to the total stress relaxation function:

$$
\begin{gathered}
S_{\mathrm{tot}}(t)=\frac{N_{b}}{k_{B} T}\left\langle\frac{1}{N_{b}^{2}} \sum_{j=1}^{N_{c}} O_{j}^{\alpha \beta}(t) \sum_{j=1}^{N_{c}} O_{j}^{\alpha \beta}(0)\right\rangle ; \\
G(t)=\frac{V}{k_{B} T}\left\langle\sigma^{\alpha \beta}(t) \sigma^{\alpha \beta}(0)\right\rangle ; \quad G(t)=\frac{1}{\alpha} S(t)
\end{gathered}
$$

where $S_{\text {tot }}(t)$ is total orientation relaxation function, $G(t)$ is the usual stress relaxation function ( $\sigma^{\alpha \beta}$ being the stress tensor), $N_{c}$ and $N_{b}=N_{c}(N-1)$ are the number of chains and bonds in the system and $\alpha$ is the stress-optical coefficient.

Figure 1 shows stress relaxation functions of two monodisperse melts and of their $50 \%$ blend, together with ori-

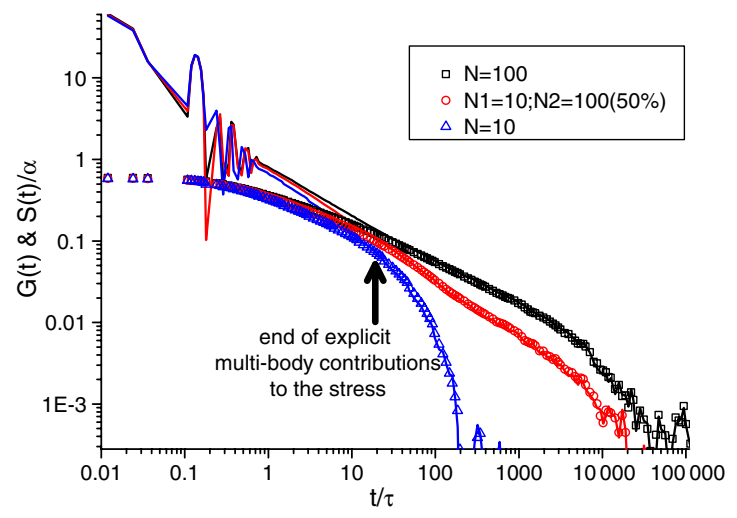

FIG. 1 (color online). Stress (lines) and orientation (open symbols) relaxation functions in monodisperse melts and their binary blends. The reason of the oscillations of stress relaxation at short times is bond length relaxation.

entation relaxation, multiplied by $1 / \alpha$. We found that $\alpha=0.0885$ for the flexible Kremer-Grest model. Three important observations are (i) indeed the two relaxation functions become proportional to each other after about 30 Lennard-Jones time units, and remain proportional to each other with accuracy better than 1\%; (ii) The stress-optical coefficient does not depend on chain lengths, and (iii) it remains the same for binary blends, in accordance with experiment. Thus, studying orientation coupling should provide useful information for the stress relaxation and rheology. Although the explicit effects of nonbonded interactions on the stress seem to be limited by short times, another important effect of nonbonded interaction, which persists at all times, is the coupling of orientational relaxation between different chains.

In order to investigate the role of these crosscorrelations, we decompose the total orientation relaxation into autocorrelations within the same chain and crosscorrelations, introducing the following notations:

$$
\begin{aligned}
C_{A B}(t) & =\frac{N_{b}}{k_{B} T} \frac{1}{\varphi_{A} \varphi_{B} N_{b}^{2}} \sum_{i \in A} \sum_{j \in B, j \neq i}\left\langle O_{i}^{\alpha \beta}(t) O_{j}^{\alpha \beta}(0)\right\rangle \\
A_{A}(t) & =\frac{N_{b}}{k_{B} T} \frac{1}{\varphi_{A} N_{b}^{2}} \sum_{i \in A}\left\langle O_{i}^{\alpha \beta}(t) O_{i}^{\alpha \beta}(0)\right\rangle,
\end{aligned}
$$

where $N_{b}$ is the total number of bonds in the system and $A$, $B$ denote different components with volume fraction $\varphi_{A}$ and $\varphi_{B}$. In these notations, total relaxation of the binary blend can be expressed as

$$
\begin{aligned}
S_{\mathrm{tot}}(t)= & \varphi_{A} A_{A}(t)+\varphi_{A}^{2} C_{A A}(t)+\varphi_{B} A_{B}(t)+\varphi_{B}^{2} C_{B B}(t) \\
& +2 \varphi_{A} \varphi_{B} C_{A B}(t) .
\end{aligned}
$$

To study these contributions in simulations, we introduced an orientation correlator for each chain, a correlator for total orientation of all chains of each type, and one correlator for the total orientation. The difference between the total and sum of autocorrelations gives different crosscorrelation terms. To exclude artefacts from the simulation 
box size, we routinely calculated $\kappa(t)$ for different box sizes, and all results reported in this Letter are within $3 \%-4 \%$ of asymptotic values for an infinite box.

Figure 2 (top) shows $S_{\text {tot }}(t)$ and total cross-correlation $C(t)$ for monodisperse melts of different lengths and for the $50 \%$ blend of $N=10$ and $N=100$ chains. The first thing to notice is that cross-correlations constitute a significant fraction of the total orientation relaxation $(50 \%$ at long times). All cross-correlations start at the same point at $t=$ 0 and are constant until $t=0.5 \tau$, and then increase a little $(15 \%)$ before starting to relax with a slope of approximately -0.5 , similar to the autocorrelations. Since $A(t)$ is decreasing faster at small times, the relative role of crosscorrelations is increasing with time. Figure 2 (bottom) shows all 5 contributions to the total orientation relaxation in binary blends. In particular, the cross-correlation between short and long chains $C_{s l}(t)$ does not decay until the relaxation time of the long chains. Instead, it becomes proportional to the total orientation relaxation function. We note that in order to predict short chain relaxation after a small step deformation using the fluctuation-dissipation theorem, one must add the short chain autocorrelation function and all cross-correlations involving the short chains [10], i.e., $\quad E_{s}(t)=\varphi_{s} A_{s}(t)+\varphi_{s}^{2} C_{s s}(t)+$ $\varphi_{s} \varphi_{l} C_{s l}(t)$. This means that although short chain $A_{s}(t)$ and $C_{s s}(t)$ relax with the short chain relaxation time, orientation of the short chains after the step deformation will not relax until the longest relaxation time of the system because of nonzero $C_{s l}(t)$. This qualitatively matches the experimental observations of Ylitalo et al. [4].

The main goal of this Letter is to describe orientational coupling with one universal function, which will capture the behavior of both monodisperse polymers and their mixtures. In particular, it should allow prediction of all cross-correlation curves on Fig. 2 (bottom) from monodisperse behavior [Fig. 2 (top)]. We find these requirements are met by the function we call a time-dependent coupling

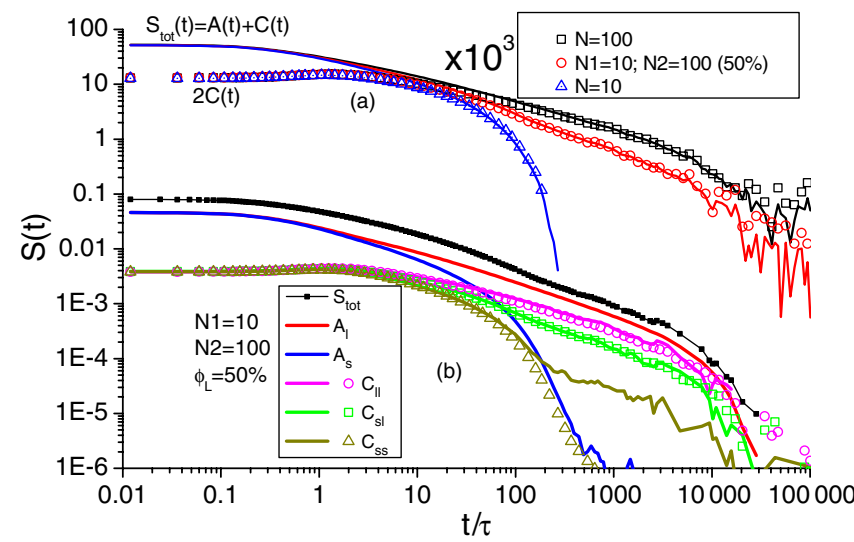

FIG. 2 (color online). Top: Total (lines) and cross-correlations (open symbol) relaxation functions (multiplied by 2). Bottom: Separate contributions (lines) in binary blends. Points show predictions of cross-correlation terms from monodisperse simulations only using Eqs. (1). parameter, $\kappa(t)=\frac{C(t)}{S_{\text {tot }}(t)}=\frac{S_{\text {tot }}(t)-A(t)}{S_{\text {tot }}(t)}$, where $C(t)$ and $A(t)$ are the sums of all cross- and all autocorrelations in the system, respectively. Clearly $\kappa(t)$ has a simple physical interpretation of relative importance of cross-correlations at different times.

Figures 3(a) and 3(b) demonstrate that $\kappa(t)$ has indeed a universal behavior, which is independent on molecular weight and blends composition. Indeed, all plots collapse onto the same master curve for several bidisperse melts (a) and for different chain lengths [(b) lines]. These two graphs also illustrate that the role of cross-correlations increases with time, rising from $12.9 \%$ at $t=0$ to about $50 \%$ at a later time. In order to find out whether entanglements play an important role in observed coupling, we simulated a system with softer nonbonded potentials $U_{n b}(r)=$ $-\frac{3}{4} u_{0}\left(r^{2}-r_{n b}^{2}\right)^{2} ; r \leq r_{n b}$ and used harmonic bonded potential $U_{b}(r)=\frac{k_{r}}{2}\left(r-r_{b}\right)^{2} ; r \leq r_{b}$, where $r_{n b}=1.6, r_{b}=$ $1.222, u_{0}=2.2, k_{r}=20$ when $k_{b}=0$. This system has approximately the same structural properties of the chains at long distances (i.e., it has exactly the same average squared internal distances $\left\langle R_{i j}^{2}\right\rangle$ and thus the same $C_{\infty}$ ), but allows easy chain crossing. We found that $\kappa(t)$ reached the same plateau (around 50\%) as the original system [points in Fig. 3(b)]. The only difference is that the terminal relaxation time is smaller than the previous one since the chains obeyed Rouse-like dynamics. Indeed we observed that orientation relaxation and mean-squared dis-
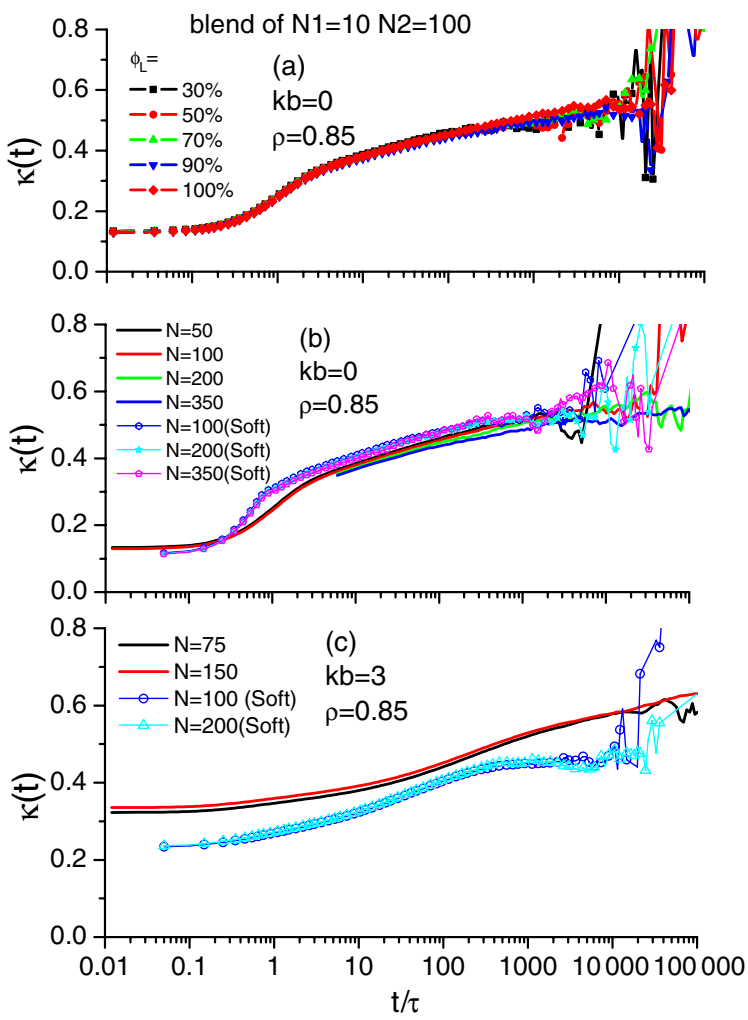

FIG. 3 (color online). Universal coupling parameter $\kappa(t)$ in binary blends with varying composition (a) and in monodisperse melts with varying molecular weights (b). 
placements were in perfect agreement with the Rouse model. The results of original entangled and soft potential simulations show that the role of entanglements in orientational coupling is negligible in this particular system, contrary to the recent argument of Deutsch and Pixley [11].

To further probe the role of entanglements we computed $\kappa(t)$ for slightly semiflexible chains with stiffness parameter $k_{b}=3$ as described in Ref. [9]. These systems have significantly smaller $N_{e}$ and thus more entanglements. The results are shown in Fig. 3(c) by lines. In this case the coupling at time zero is higher since semiflexible chains have more contacts with other chains than their fully flexible counterpart. At later times $\kappa(t)$ increases above $60 \%$. This is again compared with softer system with parameters $u_{0}=2.1, k_{r}=34$ adjusted to match internal distances $\left\langle R_{i j}^{2}\right\rangle$. The results are again very close, although some difference shows up at late time. Coupling parameter for soft systems seem to reach a plateau, whereas it continues to increase slowly for the Lennard-Jones chains. This can be interpreted as an influence of entanglements. Alternatively, one can say that in entangled system chains spend more time in contact with the same other chains, and thus become more coupled to each other. In any case, the role of entanglements seem to be minor and indirect.

The universality of time-dependent coupling parameter $\kappa(t)$ has nontrivial consequences for polymer blends. Indeed, a requirement that $\kappa(t)$ is the same in monodisperse melts and in their blend can be written as

$$
1-\kappa(t)=\frac{A_{s}(t) \varphi_{s}+A_{l}(t) \varphi_{l}}{S_{\mathrm{tot}}^{\text {blend }}}=\frac{A_{s}^{\mathrm{mono}}(t)}{S_{s}^{\mathrm{mono}}}=\frac{A_{l}^{\mathrm{mono}}(t)}{S_{l}^{\text {mono }}} .
$$

It immediately follows from here that if autocorrelation functions are the same in the mixtures as in the monodisperse systems (which is true for unentangled systems), the simple mixing rule for the total orientation relaxation $S_{\text {tot }}(t)=\varphi_{s} S_{s}^{\text {mono }}(t)+\varphi_{l} S_{l}^{\text {mono }}(t)$ is not spoiled by the orientation coupling. In entangled systems, single chain models like tube or slip-links aim to predict autocorrelation relaxation in blends. Observed orientation relaxation of short chains will be affected by the long chains via orientation coupling and tube or slip-links predictions must be modified accordingly. Universality of $\kappa(t)$ also means that one can relate different components of cross-correlation to the autocorrelation functions:

$$
\begin{gathered}
2 C_{s l}(t)=\left(S_{s}^{\text {mono }}(t)+S_{l}^{\text {mono }}(t)\right) \kappa(t) \\
C_{s s}(t)=S_{s}^{\text {mono }}(t) \kappa(t) ; \quad C_{l l}(t)=S_{l}^{\text {mono }}(t) \kappa(t) .
\end{gathered}
$$

Predictions given by these expressions are shown by open symbols in Fig. 2 (bottom). They are in perfect agreement with the lines (measured in binary blends) apart from the long-time behavior of $C_{s s}(t)$, which is affected by the secondary coupling of two short chains via an intermediate long chain. These in turn provide the response of the short component to the step deformation:

$$
E_{s}(t)=\varphi_{s} S_{s}^{\text {mono }}(t)+\frac{\varphi_{l} \varphi_{s} \kappa(t)}{2}\left(S_{l}^{\operatorname{mono}}(t)-S_{s}^{\text {mono }}(t)\right)
$$

with analogous expression for the long component. For the times longer than the relaxation time of the short component $\tau_{s}$ this equation provides a simple relation of our coupling parameter with the Doi's parameter: $\varepsilon \approx \frac{1}{2} \kappa\left(t>\tau_{s}\right)$.

In conclusion, cross-correlations between orientation tensors of different chains in polymer melt reveal rich and fascinating picture. Molecular dynamics simulations show that the role of cross-correlations in total orientational relaxation function increases with time, reaching the same amplitude as the autocorrelations. We introduced a time-dependent coupling parameter $\kappa(t)$ and found that it provides a universal description of coupling effects independent on chain length and blend composition. Instead of using static Doi's coupling parameter $\varepsilon$, which is valid only after short chain relaxation in binary blends, we can use $\kappa(t)$ to quantify coupling effects at any times in both monodisperse and bidisperse melts. Universality of $\kappa(t)$ means that we can predict any correlation function in binary blends for unentangled chains providing one knows relaxation functions in monodisperse melts and $\kappa(t)$ (also measured from monodisperse melts).

One of the main conclusions of this paper is that the orientation coupling is time dependent, with the relative role of cross-correlations increasing by a factor of about 4 towards longer times. This means that any static calculations will only be able to provide a small value $\kappa(0)$, whereas the important longtime coupling can be only obtained by dynamics calculations. This probably explains the discrepancy observed by Baljon et al. [6].

*A.Likhtman@ reading.ac.uk

[1] P. E. Rouse, J. Chem. Phys. 21, 1272 (1953).

[2] P. G. de Gennes, Scaling Concepts in Polymer Physics (Cornell University Press, Ithaca, NY, 1979).

[3] W. Merrill, M. Tirrell, J.-F. Tassin, and L. Monnerie, Macromolecules 22, 896 (1989).

[4] C. M. Ylitalo, J. A. Kornfield, G. G. Fuller, and D. S. Pearson, Macromolecules 24, 749 (1991).

[5] R. Graf, A. Heuer, and H. W. Spiess, Phys. Rev. Lett. 80, 5738 (1998).

[6] A.R.C. Baljon, G.S. Grest, and T.A. Witten, Macromolecules 28, 1835 (1995).

[7] K. Kremer and G. S. Grest, J. Chem. Phys. 92, 5057 (1990).

[8] R. Auhl, R. Everaers, G. S. Grest, K. Kremer, and S. J. Plimpton, J. Chem. Phys. 119, 12718 (2003).

[9] A.E. Likhtman, S. K. Sukumaran, and J. Ramirez, Macromolecules 40, 6748 (2007).

[10] J. Ramírez, S. K. Sukumaran, and A.E. Likhtman, J. Chem. Phys. 126, 244904 (2007).

[11] J. Deutsch and J. Pixley, Phys. Rev. E 80, 011803 (2009). 